\title{
Graded Modules as a Clean Comodule
}

\author{
Nikken Prima Puspita ${ }^{1,2}$, Indah Emilia Wijayanti ${ }^{1} \&$ Budi Surodjo $^{1}$ \\ ${ }^{1}$ Department of Mathematics, Universitas Gadjah Mada, Yogyakarta, Indonesia \\ ${ }^{2}$ Department of Mathematics. Universitas Diponegoro, Semarang, Jawa Tengah, Indonesia \\ Correspondence: Nikken Prima Puspita, Doctoral Student of Mathematics Department, Universitas Gadjah Mada, Yo- \\ gyakarta, Indonesia. E-mail: nikken.prima.p@ mail.ugm.ac.id
}

\author{
Received: September 26, 2020 Accepted: October 20, 2020 Online Published: November 11, 2020 \\ doi:10.5539/jmr.v12n6p66 URL: https://doi.org/10.5539/jmr.v12n6p66
}

\begin{abstract}
In ring and module theory, the cleanness property is well established. If any element of $R$ can be expressed as the sum of an idempotent and a unit, then $R$ is said to be a clean ring. Moreover, an $R$-module $M$ is clean if the endomorphism ring of $M$ is clean. We study the cleanness concept of coalgebra and comodules as a dualization of the cleanness in rings and modules. Let $C$ be an $R$-coalgebra and $M$ be a $C$-comodule. Since the endomorphism of $C$-comodule $M$ is a ring, $M$ is called a clean $C$-comodule if the ring of $C$-comodule endomorphisms of $M$ is clean. In Brzeziński and Wisbauer (2003), the group ring $R[G]$ is an $R$-coalgebra. Consider $M$ as an $R[G]$-comodule. In this paper, we have investigated some sufficient conditions to make $M$ a clean $R[G]$-comodule, and have shown that every $G$-graded module $M$ is a clean $R[G]$-comodule if $M$ is a clean $R$-module.
\end{abstract}

Keywords: graded modules, coalgebras, comodules, clean comodules

\section{Introduction}

Throughout, $R$ is a commutative ring with identity, $G$ is a group and $M$ is an $R$-module. Research into clean rings is an interesting topic in algebra. In their 1977 paper, Nicholson introduced the concept of a clean element. Any element $x$ of $R$ is said to be clean if $x=u+e$ with $e$ is an idempotent element and $u$ is a unit element in $R$. Moreover, if its elements are clean, then $R$ is a clean ring. Some authors, including R. B. Warfield, Jr. (1972) and Crawley and Jónnson (1964), studied and proved certain properties of exchange rings. Clean rings are a subclass of exchange rings. Another result of clean rings has already been by Han and Nicholson (2001) and Anderson and Camillo (2002). Consider the group ring $R[G]$. It is possible to study the clean element in $R[G]$ as a ring. Hence, Han and Nicholson (2001) and McGovern (2006) give the necessary and sufficient conditions for the group ring $R[G]$ to be clean.

Consider $R$ as an $R$-module over itself. The endomorphisms of $R$-module $R$ are denoted by $\left(\operatorname{End}_{R}(R)\right)$. Hence, the ring of $\operatorname{End}_{R}(R)$ is isomorphic to $R$. This implies that if $R$ is clean, then so is $E n d_{R}(R)$. The converse is also true. This condition is generalized for any $R$-module $M$. For any $R$-module $M$, we can construct the endomorphism ring of $M$ over $R$, denoted by $\operatorname{End}_{R}(M)$. The cleanness of $M$ is determined by the structure of $E n d_{R}(M)$. An $R$-module $M$ is clean if the ring of $\operatorname{End}_{R}(M)$ is clean. Camillo et al. (2006) give the conditions that make the ring of $\operatorname{End}_{R}(M)$ clean. As the main result, the paper proves that continuous modules are clean.

Now, recall the notion of comodules over a coalgebra over a field as described by Sweedler (1969). Brzeziński and Wisbauer (2003) give the generalized results for Sweedler by generalizing the ground field to any commutative ring with identity.

Throughout this paper, $(C, \Delta, \varepsilon)$ is a coassociative and counital coalgebra over $R$. Hence, the comultiplication $\Delta: C \rightarrow$ $C \otimes_{R} C$ and the counit $\varepsilon: C \rightarrow R$ are $R$-module homomorphisms which satisfy some conditions (see (Brzeziński and Wisbauer, 2003)). The $R$-module $M$ with $R$-module homomorphism $\varrho^{M}: M \rightarrow M \otimes_{R} C$, which is called a right $C$ coaction, with the properties as below (coassociative and counital):

$$
\left(I_{M} \otimes \Delta\right) \circ \varrho^{M}=\left(\varrho^{M} \otimes I_{C}\right) \circ \varrho^{M} \text { and }\left(I_{M} \circ \varepsilon\right) \circ \varrho^{M}=I_{M}
$$

is called a right $C$-comodule. In this paper, the notation $\left(M, \varrho^{M}\right)$ is a right $C$-comodule $M$ with $C$-right coaction $\varrho^{M}$. In module theory, we are already familiar with homomorphism between two modules. In the comodule theory, we have a similar concept to module homomorphism, i.e., the comodule morphism. Let $\left(M, \varrho^{M}\right)$ and $\left(N, \varrho^{N}\right)$ be right $C$-comodule. 
A right $C$-comodule morphism $f: M \rightarrow N$ is an $R$-module homomorphism which satisfies the following condition:

$$
\varrho^{N} \circ f=\left(f \otimes I_{C}\right) \circ \varrho^{M}
$$

with $I_{C}$ is the identity map of $C$. The set of all right $C$-comodule morphisms from $M$ to $N$ is denoted by $H_{o m}{ }^{C}(M, N)$. Moreover, the category of $C$-comodules is denoted by $M^{C}$. If $M=N$, then the endomorphism of $C$-comodule $M$, i.e., $E n d^{C}(M)$ is a ring over the addition and composition product.

From the $R$-coalgebra $C$ we can construct the dual $R$-algebra $C^{*}=\operatorname{Hom}_{R}(C, R)$ by defining the convolution product as below:

$$
f_{1} * f_{2}=\mu \circ\left(f_{1} \otimes f_{2}\right) \circ \Delta,
$$

for any $f_{1}, f_{2} \in C^{*}, \mu$ is the multiplication of $R$ and $\Delta$ is the comultiplication of $R$-coalgebra $C$. In Brzeziński and Wisbauer (2003), $\left(C^{*},+, *\right)$ is a dual $R$-algebra. Moreover, any right $C$-comodule $M$ can be considering as a left $C^{*}$-module by the following scalar multiplication

$$
\rightarrow: C^{*} \otimes_{R} M \rightarrow M, g \otimes a \mapsto\left(I_{M} \otimes g\right) \circ \varrho^{M}(a)
$$

By Sweedler's notation, for any $g \otimes a \in C^{*} \otimes_{R} M,\left(I_{M} \otimes g\right) \circ \varrho^{M}(a)=\sum a_{0} g\left(a_{1}\right) \in M$. On the other hand, every right $C$-comodule morphism is a left $C^{*}$-module morphism such that $E n d^{C}(M)$ is a subring of $C^{*} E n d(M)$. The relation between them is isomorphic if and only if $C$ is locally projective as an $R$-module (see the $\alpha$-condition (Brzeziński and Wisbauer, 2003)). Thus, if $C$ is a locally projective $R$-module, $E n d^{C}(M) \simeq_{C^{*}} E n d(M)$. In this paper, the notion of clean comodules depends on the structure of $\operatorname{End}^{C}(M)$.

The graded ring $R[G]$ is an $R$-coalgebra, by taking the comultiplication $\Delta: R[G] \rightarrow R[G] \otimes_{R} R[G], g \rightarrow g \otimes g$ and the counit $\varepsilon: R[G] \rightarrow R, g \mapsto 1$. When we have graded modules in comodules, it can be considered a comodule over the coalgebra $R[G]$. Our paper will focus on graded modules as the comodules structure. We recall the definition of graded rings and graded modules, which are referred to by Natasescu and Oystaeyen in their 1982 and 2004 papers.

Definition 1.1(Natasescu and Oystaeyen, 2004) Let $G$ be a group (or semigroup) with identity element e. A ring $R$ is called a $G$-graded ring if there is a family of additive subgroups of $R$ i.e., $\left\{R_{\sigma} \mid \sigma \in G\right\}$ such that $R=\oplus_{\sigma \in G} R_{\sigma}$ and $R_{\sigma} R_{\lambda} \subseteq R_{\sigma \lambda}$, for every $\sigma, \lambda \in G$. The $G$-graded ring $R$ is strongly if $R_{\sigma} R_{\lambda}=R_{\sigma \lambda}$ for all $\sigma, \lambda \in G$.

Let $R=\oplus_{\sigma \in G} R_{\sigma}$ and $S=\oplus_{\sigma \in G} S_{\sigma}$ be two $G$-graded rings. A ring homomorphism $f: R \rightarrow S$ is called a graded homomorphism if $f\left(r_{\sigma}\right)=s_{\sigma}$ for all $\sigma \in G$.

For any group $G$, the category of $G$-graded rings is denoted by $G-R I N G$. The objects of $G-R I N G$ are the class of $G$-graded rings and the set of all graded homomorphisms. If $G=\{e\}$, then $G-R I N G$ and the category of rings coincide.

Definition 1.2 (Natasescu and Oystaeyen, 2004) Let $G$ be a group and $R=\oplus_{\sigma \in G} R_{\sigma}$ a graded ring of type G. A (left) $G$-graded module is a (left) $R$-module $M$ such that $M=\oplus_{x \in G} M_{x}$, where every $M_{x}$ is an additive subgroup of $M$ and for every $\sigma$ and $x$ in $G, R_{\sigma} M_{x} \subseteq M_{\sigma x}$.

Definition 1.3(Natasescu and Oystaeyen, 1982) Let $G$ be a group and $R=\oplus_{\sigma \in G} R_{\sigma}$ a graded ring of type $G$. Let $M$ and $N$ be $G$-graded modules over $R$. An R-module homomorphism $f: M \rightarrow N$ is called a graded homomorphism $R$-module if $f\left(M_{\sigma}\right) \subseteq N_{\sigma}$ for every $\sigma \in G$.

Let $R$ be a $G$-graded ring. The category of $G$-graded (left) modules over $R$ is denoted by ${ }_{g r} \mathbf{M}$. The set of all morphisms from $M$ to $N$ in the category of ${ }_{g r} \mathbf{M}$ is denoted by $\operatorname{Hom}_{g r}(M, N)$. Hence, the category ${ }_{g r} \mathbf{M}$ is a subcategory of $R$-modules ${ }_{R} \mathbf{M}$ and the set of graded endomorphisms of $M$, denoted by $\operatorname{End}_{g r}(M)$, is a subring of $E n d_{R}(M)$.

Let $G$ be a group and $\left(R[G], \Delta_{1}, \varepsilon_{1}\right)$ be an $R$-coalgebra with comultiplication and counit as defined in Brzeziński and Wisbauer (2003). We have the following relationship:

Theorem 1.4(Brzeziński and Wisbauer, 2003) Let $G$ be a group (semigroup). Considering a ring $R$ with the trivial grading, an $R$-module $M$ is $G$-graded if and only if it is an $R[G]$-comodule.

Theorem 1.4 means that when we have an arbitrary graded module over a $\operatorname{ring} R$ with type $G$, we always construct a comodule over coalgebra $R[G]$. In this paper, we will focus on this fact. For any (right) $C$-comodule $M$, the comodule endomorphism $\left(E n d^{C}(M),+, \circ\right)$ is a ring with addition and composition operations. We are going to use this fact to define the notion of a clean comodule. On the other hand, every graded module over $R$ with type $G$ is an $R[G]$-comodule. Using some properties of comodule categories, module categories, and graded modules, we obtain a condition that makes $R[G]-$ comodules clean comodules. Our main result is that if $M$ is a clean $R$-module, then the $G$-graded module $M$ over $R$ is a clean comodule over an $R$-coalgebra $R[G]$. 


\section{Results}

The concept of clean comodules has become to the concept of clean modules. Whether any $R$-module $M$ is clean or not clean depends on the structure of the endomorphism ring of $\operatorname{End}_{R}(M)$. Hence, for any $M \in \mathbf{M}^{C}$, we also have the fact that the endomorphism of $C$-comodule, i.e., $\left(E n d^{C}(M),+, \circ\right)$ is a ring with unity. This fact is used to define a clean concept in a comodule structure. Based on the definition of clean modules, we introduce clean comodules.

Definition 2.1 A C-comodule $M$ is called a clean $C$-comodule if the endomorphism ring of $E n d^{C}(M)$ is clean

The clean coalgebra is defined as a special case of clean comodules. Since any $R$-coalgebra $C$ is a $C$-comodule, $C$ is said to be clean if $C$ is clean as a comodule over itself or the endomorphism ring of $E n d^{C}(C)$ is clean. In (Brzeziński and Wisbauer, 2003), if $C$ is a locally projective module, then we have a special condition related to the relationship between $\operatorname{End} d^{C}(M)$ and $C^{*} \operatorname{End}(M)$. Hence, $C^{*} \operatorname{End}(M) \simeq E n d^{C}(M)$, Moreover, for $R$-coalgebra $C, C$ is clean if and only if $C^{*} \operatorname{End}(M) \simeq \operatorname{End}^{C}(M) \simeq C^{*}$.

Any ring $R$ can be considered a trivial coalgebra by comultiplication $\Delta: R \rightarrow R \otimes_{R} R, r \mapsto r \otimes r$ and counit $\varepsilon: R \rightarrow R, r \mapsto 1$. Hence, the ring $\operatorname{End}^{R}(R, R) \simeq R$ by mapping $f \mapsto f(1)$. Consequently, if $R$ is a clean ring, then $R$ is also clean as an $R$ coalgebra. For another example, we offer the following case.

Example 2.2 Let the ring $\mathbb{Z}_{5}$. Consider $\mathbb{Z}_{5}$ a $\mathbb{Z}_{5}$-coalgebra by the (coassociative) comultiplication as below:

$$
\Delta: \mathbb{Z}_{5} \rightarrow \mathbb{Z}_{5} \otimes_{\mathbb{Z}_{5}} \mathbb{Z}_{5}, a \mapsto a \otimes 1+1 \otimes a .
$$

Since $\mathbb{Z}_{5}$ is a field, then it is a locally projective module over itself. Consequently, the $\operatorname{End} d^{\mathbb{Z}_{5}}\left(\mathbb{Z}_{5}\right) \simeq_{\mathbb{Z}_{5}^{*}} \operatorname{End}\left(\mathbb{Z}_{5}\right) \simeq \mathbb{Z}_{5}^{*}$ (Brzeziński and Wisbauer, 2003). Hence, $\mathbb{Z}_{5}^{*}=E n d_{\mathbb{Z}_{5}}\left(\mathbb{Z}_{5}\right)=\left\{g_{0}, g_{1}, g_{2}, g_{3}, g_{4}\right\}$ where $g_{0}$ is a zero map, $g_{1}$ is an identity map of $\mathbb{Z}_{5}$. Otherwise,

$$
\begin{gathered}
g_{2}(a)= \begin{cases}\overline{0} & \text { if } a=\overline{0} \\
\overline{2} & \text { if } a=\overline{1} \\
\overline{4} & \text { if } a=\overline{2} \\
\overline{1} & \text { if } a=\overline{3} \\
\overline{3} & \text { if } a=\overline{4}\end{cases} \\
g_{3}(a)= \begin{cases}\overline{0} & \text { if } a=\overline{0} \\
\overline{3} & \text { if } a=\overline{1} \\
\overline{1} & \text { if } a=\overline{2} \\
\overline{4} & \text { if } a=\overline{3} \\
\overline{2} & \text { if } a=\overline{4}\end{cases} \\
g_{4}(a)= \begin{cases}\overline{0} & \text { if } a=\overline{0} \\
\overline{4} & \text { if } a=\overline{1} \\
\overline{3} & \text { if } a=\overline{2} \\
\overline{2} & \text { if } a=\overline{3} \\
\overline{1} & \text { if } a=\overline{4}\end{cases}
\end{gathered}
$$

As a dual $R$-algebra and by using the convolution product of $*$, i.e.,

$$
g_{i} * g_{j}=\left(g_{i} \otimes g_{j}\right) \circ \Delta,
$$

for any $g_{i}, g_{j} \in \mathbb{Z}_{5}^{*}$ and $i, j=1,2,3,4,5$. We have the following table.

Table 2.1. The Result of Convolution Product in $\mathbb{Z}_{5}^{*}$

\begin{tabular}{|c|lllll|}
\hline$*$ & $g_{0}$ & $g_{1}$ & $g_{2}$ & $g_{3}$ & $g_{4}$ \\
\hline$g_{0}$ & $g_{0}$ & $g_{0}$ & $g_{0}$ & $g_{0}$ & $g_{0}$ \\
$g_{1}$ & $g_{0}$ & $g_{2}$ & $g_{4}$ & $g_{1}$ & $g_{3}$ \\
$g_{2}$ & $g_{0}$ & $g_{1}$ & $g_{3}$ & $g_{2}$ & $g_{4}$ \\
$g_{3}$ & $g_{0}$ & $g_{1}$ & $g_{2}$ & $g_{3}$ & $g_{4}$ \\
$g_{4}$ & $g_{0}$ & $g_{3}$ & $g_{1}$ & $g_{4}$ & $g_{2}$ \\
\hline
\end{tabular}


Based on the result of Table 2.1, we can see that the multiplicative identity of $\mathbb{Z}_{5}^{*}$ is $g_{3}$ and any non-zero element of $\mathbb{Z}_{5}^{*}$ is a unit. Thus, the ring $\mathbb{Z}_{5}^{*}$ is a field. Clearly, any field is a clean ring. This means that the ring $E n d^{\mathbb{Z}_{5}}\left(\mathbb{Z}_{5}\right)$ which is isomorphic with the ring $\mathbb{Z}_{5}^{*}$ is clean. This fact implies that the $\mathbb{Z}_{5}$-coalgebra $\left(\mathbb{Z}_{5}, \Delta, \varepsilon\right)$ is clean.

In this paper we will focus on investigating the cleanness of comodule $M$ over $R$-coalgebra $R[G]$. That is, we will concentrate on proving the endomorphism ring $\operatorname{End} d^{R[G]}(M)$ to be clean. Throughout, ring $R$ is a $G$-graded ring, $\mathbf{M}^{R[G]}$ is the category of $R[G]$-comodules. Defining the following map

$$
F:{ }_{g r} \mathbf{M} \rightarrow \mathbf{M}^{R[G]} \text {, where } M \mapsto M, f \mapsto f,
$$

we obtain an equivalence functor as we find in the following

Lemma 2.3 Let $G$ be a group and $R$ be a $G$-graded ring by trivial grading. The map $F$ in Equation (8) is an equivalence functor.

Proof Based on Theorem 1.4, for all $M$ in ${ }_{g r} \mathbf{M}$ we get $F(M)=M$ in $\mathbf{M}^{R[G]}$. Furthermore, take any morphism $f: M \rightarrow N$ in the category $\mathbf{M}^{R[G]}$. By considering $\left(M, \varrho^{M}\right)$ and $\left(N, \varrho^{N}\right)$ as $R[G]$-comodules we prove that $\varrho^{N} \circ f=\left(f \otimes I_{R[G]}\right) \circ \varrho^{M}$.

For any $m=\sum_{g \in G} m_{g} \in M$ we have

$$
\begin{aligned}
\left(f \otimes I_{R[G]}\right) \circ \varrho^{M}(m) & =\left(f \otimes I_{R[G]}\right) \circ \varrho^{M}\left(\sum_{g \in G} m_{g}\right) \\
& =\left(f \otimes I_{R[G]}\right)\left(\sum_{g \in G} m_{g} \otimes g\right) \\
& =\sum_{g \in G} f\left(m_{g}\right) \otimes g \\
& =\varrho^{N}\left(\sum_{g \in G} f\left(m_{g}\right)\right) \\
& =\left(\varrho^{N} \circ f\right)\left(\sum_{g \in G} m_{g}\right)\left(\text { since } f \in \operatorname{Hom}_{g r}(M, N)\right) \\
& =\left(\varrho^{N} \circ f\right)(m)
\end{aligned}
$$

Hence, for any $f$ in $\operatorname{Hom}_{g r}(M, N)$ we have $F(f)$ in $\operatorname{Hom}^{R[G]}(M, N)$. In particular, for any $G$-graded homomorphism $f$ we can consider this $f$ as a $C$-comodule morphism. Thus, we conclude that the functor $F$ is well-defined. Moreover, it is easy to prove that $F$ is a covariant functor.

Furthermore, we prove that $F$ is an equivalence functor. For any $M, N$ in ${ }_{g r} \mathbf{M}$,

$$
\begin{aligned}
F_{M, N}=\operatorname{Hom}_{g r}(M, N) & \rightarrow \operatorname{Hom}^{R[G]}(F(M), F(N))=\operatorname{Hom}^{R[G]}(M, N), \\
f & \mapsto f .
\end{aligned}
$$

It is obvious that $F_{M, N}$ is injective, so $F$ is faithful.

Now suppose that $\beta$ in $\operatorname{Hom}^{R[G]}(M, N)$. Put $m=\sum_{g \in G} m_{g} \in M$, we obtain

$$
\begin{aligned}
\beta(m) & =\beta\left(\sum_{g \in G} m_{g}\right)=\sum_{g \in G} \beta\left(m_{g}\right) \\
& =\sum_{g \in G} n_{g} \text { where } n_{g}=\beta\left(m_{g}\right) \text { for all } g \in G .
\end{aligned}
$$

Since for all $g \in G, \beta\left(m_{g}\right)=n_{g}, \beta$ is a $G$-graded homomorphism. Therefore, for any $\beta \in \operatorname{Hom}^{R[G]}(M, N)$, there exist $\beta \in \operatorname{Hom}_{g r}(M, N)$, such that $F(\beta)=\beta$. Thus $F$ is full.

Based on Theorem 1.4, functor $F$ is essentially surjective. Hence, $F$ is a equivalence functor and moreover, the category ${ }_{g r} \mathbf{M} \simeq \mathbf{M}^{R[G]}$.

Lemma 2.3 implies that $E n d^{R[G]}(M) \simeq \operatorname{End}_{g r}(M) \subseteq \operatorname{End}_{R}(M)$. In order to obtain some necessary and sufficient conditions of cleanness in $R[G]$-comodules, we need to observe the relationship of endomorphisms of $M$ in categories ${ }_{R} \mathbf{M},{ }_{g r} \mathbf{M}$, and $\mathbf{M}^{R[G]}$. 
Now we need to understand how the functor $F$ preserves the cleanness property in $\operatorname{End}_{g r}(M)$ to $\operatorname{End} d^{R[G]}(M)$.

Lemma 2.4 Every clean element on the endomorphism ring of G-graded module $M$ over $R\left(\operatorname{or}_{\operatorname{End}}(M)\right)$ is also a clean element in $\operatorname{End}^{R[G]}(M)$.

Proof Suppose that $f$ is a clean element in $\operatorname{End}_{g r}(M)$, i.e., $f=u+e$ where $u$ is a unit and $e$ is idempotent in $\operatorname{End}_{g r}(M)$. Based on definition $F$ in Equation (8), it is easy to prove that $F$ preserves the addition operation, unit and idempotent property from category ${ }_{g r} \mathbf{M}$ to $M^{R[G]}$, such that $F(f)=F(u+e)=F(u)+F(e)=u+e$. Hence, we now consider $F(u)=u$ (resp. $F(e)=e$ ) as a unit (resp. an idempotent) element in $E n d^{R[G]}(M)$ (see Lemma 2.3).

Thus, there exist a unit $F(u)=u$ and an idempotent $F(e)=e$ in $E n d^{R[G]}(M)$ such that $f=u+e$ as a morphism of $R[G]$-comodule. This means $f$ is a clean element in $\operatorname{End}^{R[G]}(M)$.

Lemma 2.4 shows that the cleanness of $\operatorname{End}_{g r}(M)$ is preserved in $E n d^{R[G]}(M)$. Furthermore, we investigate the connection between a clean element in $\operatorname{End}_{g r}(M)$ and $\operatorname{End}_{R}(M)$.

According to Theorem I.3.4 in Natasescu and Oystaeyen (1982), a strongly graded ring can be characterized by certain functors. If $R$ is a $G$-graded ring, then $R$ is a strongly graded ring if and only if functors

$$
\begin{aligned}
R \otimes_{R_{e}}-: R_{e} \mathbf{M} & \rightarrow{ }_{g r} \mathbf{M} \\
M & \mapsto R \otimes_{R_{e}} M=\oplus_{\sigma \in G} R_{\sigma} \otimes_{R_{e}} M \\
f: M \rightarrow N & \mapsto f^{\prime}: R \otimes_{R_{e}} M \rightarrow R \otimes_{R_{e}} N
\end{aligned}
$$

and

$$
\begin{aligned}
(-)_{e}:{ }_{g r} \mathbf{M} & \rightarrow{ }_{R_{e}} \mathbf{M} \\
M & \mapsto M_{e} \\
f: M \rightarrow N & \mapsto \hat{f}: M_{e} \rightarrow N_{e}
\end{aligned}
$$

are equivalent.

Therefore, if $R$ has trivial grading (i.e., $R_{e}=R$ ), then the functor $(-)_{e}: g r \mathbf{M} \rightarrow_{R} \mathbf{M}$ equivalent to the functor $R \otimes_{R}-:_{R}$ $\mathbf{M} \rightarrow g r$ M.

In general, a $G$-graded module over $R$ is not necessarily a clean $R$-module. As an example, $\mathbb{Z}$ is a $\mathbb{Z}$-graded module by trivial grading. However, we know that $\mathbb{Z}$ is not a clean module over itself. Based on these reasons and some related properties between ${ }_{R} \mathbf{M},{ }_{g r} \mathbf{M}$ and $\mathbf{M}^{R[G]}$, we obtain the following theorem.

Theorem 2.5 Let $R$ be a ring, $G$ be a group and $M$ be a clean $R$-module. Consider $R$ as a $G$-graded ring by trivial grading. If $M$ is a $G$-graded module over $R$, then $M$ is a clean $R[G]$-comodule.

Proof Based on Brzeziński and Wisbauer (2003), $M$ is an $R[G]$-comodule. Since $R$ is a $G$-graded ring by trivial grading, $R_{e}=R$ and $R_{g}=0$ for all $g \neq e$. This implies ${ }_{g r} \mathbf{M} \simeq_{R e} \mathbf{M}={ }_{R} \mathbf{M}$.

For the first case, we need to prove that $\operatorname{End}_{g r}(M)$ is also a clean ring. Take any $f \in \operatorname{End} g r(M) \subseteq \operatorname{End}_{R}(M)$. Since $M$ is a clean $R$-module, $f=u+e$ for a unit $u$ and an idempotent $e$ in $E n d_{R}(M)$. Recall the functor $R \otimes_{R_{e}}-$ with $R_{e}=R$. For any morphism $f: M \rightarrow N$ in ${ }_{R} \mathbf{M}$ we get:

$$
\left(R \otimes_{R}-\right):{ }_{R} \mathbf{M} \rightarrow{ }_{g r} \mathbf{M}
$$

For any $f: M \rightarrow N \in{ }_{R} \mathbf{M},\left(R \otimes_{R}-\right)(f)=I_{R} \otimes f=f^{\prime}: R \otimes_{R} M \rightarrow R \otimes_{R} N$. Therefore, for every $f=u+e$ in $\operatorname{End}_{R}(M)$ we have

$$
\begin{aligned}
\left(R \otimes_{R}-\right)(f) & =I_{R} \otimes_{R} f \\
& =I_{R} \otimes(u+e) \\
& =\left(I_{R} \otimes u\right)+\left(I_{R} \otimes e\right) \\
& =u^{\prime}+e^{\prime} .
\end{aligned}
$$

1. Since $u$ is a unit in $\operatorname{End}_{R}(M)$, there is $u^{-1}$ in $\operatorname{End}_{R}(M)$ such that $u \circ u^{-1}=u^{-1} \circ u=I_{M}$ in $\operatorname{End}_{R}(M)$. Hence, there 
exist $\left(u^{-1}\right)^{\prime}=R \otimes_{R}\left(u^{-1}\right)=I_{R} \otimes u^{-1}$ in $\operatorname{End}_{g r}(M)$ such that for any $\sum_{g \in G} r_{g} \otimes_{R} m \in{ }_{g r} \mathbf{M}$, we have

$$
\begin{aligned}
\left(I_{R} \otimes u^{-1}\right) \circ\left(I_{R} \otimes u\right)\left(\sum_{g \in G} r_{g} \otimes_{R} m\right) & =\left(I_{R} \otimes u^{-1}\right)\left(\sum_{g \in G} r_{g} \otimes_{R} u(m)\right) \\
& =\sum_{g \in G} r_{g} \otimes_{R} u^{-1} u(m) \\
& =\sum_{g \in G} r_{g} \otimes_{R} m \\
& =I_{R \otimes_{R} M}\left(\sum_{g \in G} r_{g} \otimes_{R} m\right) \\
& =I_{M}\left(\sum_{g \in G} r_{g} \otimes_{R} m\right)\left(\text { since } R \otimes_{R} M \simeq M\right) .
\end{aligned}
$$

Thus, $\left(I_{R} \otimes u^{-1}\right) \circ\left(I_{R} \otimes u\right)=I_{R \otimes_{R} M} \simeq I_{M}$. Similarly we have $\left(I_{R} \otimes u\right) \circ\left(I_{R} \otimes u^{-1}\right)=I_{R \otimes_{R} M} \simeq I_{M}$. This implies $\left(I_{R} \otimes u\right)$ is a unit in $E n d_{g r} M$.

2. Moreover, for $f^{\prime}=I_{R} \otimes f$ we want to see that $e^{\prime}=I_{R} \otimes e$ is an idempotent element in $\operatorname{End}_{g r}(M)$. Take any $\sum_{g \in G} r_{g} \otimes_{R} m$ in ${ }_{g r} \mathbf{M}$, and we have

$$
\begin{aligned}
e^{\prime} e^{\prime}\left(\sum_{g \in G} r_{g} \otimes_{R} m\right) & =\left(I_{R} \otimes e\right) \circ\left(I_{R} \otimes e\right)\left(\sum_{g \in G} r_{g} \otimes_{R} m\right) \\
& =\left(I_{R} \otimes e\right)\left(\sum I_{R}\left(\sum_{g \in G} r_{g}\right) \otimes e(m)\right) \\
& =\left(I_{R} \otimes e\right)\left(\sum_{g \in G} r_{g} \otimes e(m)\right) \\
& =\sum_{g \in G} r_{g} \otimes e e(m) \\
& =\sum_{g \in G} r_{g} \otimes_{R} e(m) \\
& =\left(I_{R} \otimes e\right)\left(\sum_{g \in G} r_{g} \otimes_{R} m\right)
\end{aligned}
$$

Hence, $e^{\prime}=I_{R} \otimes_{R} e$ is an idempotent element in $\operatorname{End} g r(M)$.

From point (1) and (2), for any $f$ in $\operatorname{End}_{g r}(M) \subseteq \operatorname{End}_{R}(M)$, where $f=u+e$ in $\operatorname{End}_{R}(M)$, there exist $u^{\prime}=I_{R} \otimes u$ a unit in $E n d_{g r}(M)$ and $e^{\prime}=I_{R} \otimes e$ an idempotent element in $\operatorname{End}_{g r}(M)$ such that $f \approx f^{\prime}=I_{R} \otimes_{R} f=I_{R} \otimes_{R} u+I_{R} \otimes_{R} e=u^{\prime}+e^{\prime}$, or $f \approx f^{\prime}$ is a clean element in $E n d_{g r}(M)$. Consequently, $E n d_{g r}(M)$ is a clean ring. This means that if the $E n d_{R}(M)$ is a clean ring, then $\operatorname{End}_{g r}(M)$ is also a clean ring. Our objective is to prove that the $E n d^{R[G]}(M)$ is a clean ring. We derive the conclusion from previous facts. Based on Lemma, we have

$$
\operatorname{End}^{R[G]}(M) \simeq{ }_{g r} \operatorname{End}(M) .
$$

As we have assumed, since $M$ is a clean $R$-module, $\operatorname{End}_{R}(M)$ is a clean ring. This fact implies the $\operatorname{ring}{ }_{g r} \operatorname{End}(M)$ is also clean. Moreover, by isomorphisms in Equation 9 and clean properties in Lemma 2.4, we have the result that the endomorphism ring $\operatorname{En} d^{R[G]}(M)$ is clean. Consequently, $M$ is a clean $R[G]$-comodule.

Example 2.6 Let $G$ be a group and $M$ an injective $R$-module. If $R$ is a $G$-graded ring by trivial grading, then $M$ is a $G$-graded $R$-module; moreover, $M$ is a clean $R[G]$-comodule since any injective $R$-module is clean (Camillo et al., 2006).

The converse of Theorem 2.5 is also true. By functor $F$ in Equation (8) if $M$ is an $R[G]$-comodule, then $M$ is a $G$-graded module over $R$. Therefore, we have the following theorem.

Theorem 2.7 Let $R$ be a ring, $G$ a group. Consider $R$ as a $G$-graded ring by trivial grading. If $M$ is a clean $R[G]$-comodule, then $M$ is a $G$-graded $R$-module and clean as an R-module.

Proof It is obvious that if $M$ is an $R[G]$-comodule, then $M$ is a $G$-graded module over $R$. Hence, $M$ is an $R$-module. Suppose that $M$ is clean as an $R[G]$-comodule. That is, $E n d^{R[G]}(M)$ is a clean ring. By Lemma 2.3 and Lemma 2.4 $E n d^{R[G]}(M) \simeq E n d_{g r}(M)$ is a clean ring. Moreover, by functor $R \otimes_{R_{e}}-$ with $R_{e}=R$ we have that ${ }_{g r} \mathbf{M} \simeq_{R} \mathbf{M}$. For any 
$f \in \operatorname{End}_{g r}(M), f \in \operatorname{End}_{R}(M)$. Consequently, if $f$ is a clean element in $\operatorname{End}_{g r}(M)$, then $f$ is also clean as an element of $\operatorname{End}_{R}(M)$. This means $M$ is a clean $R$-module.

Let $R$ be a $G$-graded ring by trivial grading or $R_{e}=R$. By the equivalence functors of $F:_{g r} \mathbf{M} \rightarrow \mathbf{M}^{R[G]}$ and $R \otimes_{R_{e}}-:_{R_{e}}$ $\mathbf{M} \rightarrow_{g r} \mathbf{M}$, we have the result that the category of $R_{e} \mathbf{M} \simeq_{g r} \mathbf{M} \simeq \mathbf{M}^{R[G]}$ with $R_{e}=R$. Thus, Theorem 2.5 and Theorem 2.7 show that the cleanness property of each category can be inherited by the other. Consequently, we can adopt properties of clean modules to the clean $R[G]$-comodules.

For any $C$-comodule $M$, the endomorphism of $C$-comodule or $E n d^{C}(M)$ is a ring over the addition and composition function. We introduced clean comodules by following certain concepts of clean modules theory. A $C$-comodule $M$ is clean if $E n d^{C}(M)$ is a clean ring. Consider the group ring $R[G]$ as an $R$-coalgebra. This paper investigates the cleanness of comodules over $R$-coalgebra $R[G]$. Using some category theory, the comodule $M$ over $R[G]$ is determined by the structure of $R$-module $M$.

\section{Discussion}

The study of cleanness in algebra structures is also applied to near-ring structures (see Hashemi, E. et al., 2020). The module over a near-ring was studied by Beidleman (1964). We can improve our results to study the cleanness of the module over a near-ring. For future research, Theorem 2.5 can be generalized by changing the clean $R$-module $M$ to become a clean module over a near-ring. We conclude this paper with the question: Is the statement in Theorem 2.5 still valid if we assume that $M$ is a clean module over a near-ring?

\section{Acknowledgements}

This research is supported by Doctoral Research Grant, Directory of Higher Education, The Ministry of Education and Culture, Indonesia, 2019-2020. We would like to thank Prof. Alexander Zimmermann (Univ. Picardie Jules Verne, Amiens, France) for his warm hospitality when the firs author was doing visiting research in Amiens under his supervision.

\section{References}

Anderson, D. D., \& Camillo, V. P. (2002) Commutative rings whose elements are a sum of a unit and idempotent, Comm. Algebra, 30, 3327-3336. https://doi.org/10.1081/AGB-120004490

Beidleman, J. C. (1964). On Near-Rings and Near-Rings Modules. Mathematics Thesis. University Microfilms, Inc., Ann Arbor, Michigan.

Brzeziński, T., \& Wisbauer, R. (2003). Corings and Comodules. Cambridge University Press, United Kingdom. https://doi.org/10.1017/CBO9780511546495

Camillo, V. P., Khurana, D., Lam, T. Y., Nicholson, W. K., \& Zhou, Y. (2006) Continuous modules are clean. J. Algebra, 304, 94-111. https://doi.org/10.1016/j.jalgebra.2006.06.032

Crawley, P., \& Jónnson, B. (1964). Refinements for infinite direct decompositions of algebraic systems. Pacific J. Math, 14, 797-855. https://doi.org/10.2140/pjm.1964.14.797

Han, J., \& Nicholson, W. K. (2001). Extension of clean rings. Comm. Algebra, 29, 258-2595. https://doi.org/10.1081/AGB100002409

Hashemi, E., Shokuhifar, F., \& Alhevaz, A. (2020). On clean and regular elements of near-ring of skew polynomials. Communications In Algebra, 48, 2575-2590. https://doi.org/10.1080/00927872.2020.1721516

McGovern, W. W. (2006). Characterization of commutative clean rings. Int. J. Math. Game Theory Algebra, 15(40), 403-413.

Natasescu, C., \& Oystaeyen, F. V. (1982). Graded Ring Theory. North-Holland Amsterdam-New York.

Natasescu, C., \& Oystaeyen, F. V. (2004). Methods of Graded Rings, Springer-Verlag Berlin Heidelberg. https://doi.org/10.1007/b94904

Nicholson, W. K. (1977). Lifting idempotents and exchange rings. Trans. Amer. Math. Soc, 229, 269-278. https://doi.org/10.1090/S0002-9947-1977-0439876-2

Sweedler, M. E. (1969). Hopf Algebra, Mathematics Lecture Note Series. W.A. Benjamins, Inc.

Warfield, J. R. B. (1972). Exchange rings and decompositions of modules. Math. Ann, 199, 31-36. https://doi.org/10.1007/BF01419573 


\section{Copyrights}

Copyright for this article is retained by the author(s), with first publication rights granted to the journal.

This is an open-access article distributed under the terms and conditions of the Creative Commons Attribution license (http://creativecommons.org/licenses/by/4.0/). 